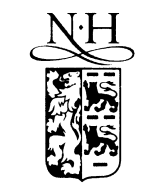

ELSEVIER

Nuclear Instruments and Methods in Physics Research B 154 (1999) 25-31

Beam Interactions

with Materials \& Atoms

www.elsevier.nl/locate/nimb

\title{
Analytical approaches to the fragmentation of few-body Coulomb systems
}

\author{
J. Berakdar ${ }^{1}$ \\ Max-Planck Institut für Mikrostrukturphysik, Weinberg 2, 06120 Halle, Germany
}

\begin{abstract}
This work reviews recent progress in the analytical treatment of correlated few-body Coulomb continuum systems. Appropriate curvilinear coordinates for the representation of such systems are discussed and their usefulness is demonstrated. Approximate methods for dealing with the short-range dynamics are briefly discussed. (C) 1999 Elsevier Science B.V. All rights reserved.
\end{abstract}

PACS: 34.80.Dp; 34.10.+x; 25.10.+s

Keywords: Many-body problem; Coulomb systems; Scattering theory; Electron-impact ionization

\section{Introduction}

The difficulties in the theoretical treatment of dissociative reactions involving few charged particles stem from the inherent non-separability of many-body interacting systems. In addition, such treatments are hampered by the infinite range of the Coulomb interaction that precludes free asymptotic states and hence limits seriously the applicability of standard methods of scattering theory. Thus, in recent years, much efforts have been focused towards a direct (approximative) solutions of the Schrödinger equation in the fragmentation channel without going through the procedures of scattering theory [1-11]. Such solu-

\footnotetext{
${ }^{1}$ Fax: +49-345-55-11-22-3; e-mail: jber@mpi-halle.de
}

tions, though cumbersome to derive, yield a direct insight into the momentum- and configurationspace distributions of the fragments. As shown below, the essential point in deriving useful expressions for the eigenstates of the many-body Hamiltonian is the choice of appropriate coordinates that already contain some characteristics of the Coulomb forces.

\section{Two-body Coulomb scattering}

To sense the "natural" coordinates for fragmentation processes involving Coulomb potentials it is instructive to consider the non-relativistic scattering of two charge particles with charges $Z_{1}$ and $Z_{2}$. The Schrödinger equation describing the motion in the two-particle relative coordinate $\mathbf{r}$ is (atomic units are used throughout) 


$$
\left[-\frac{1}{2 \mu} \Delta+\frac{Z_{1} Z_{2}}{r}-E\right] \Psi_{\mathbf{k}}(\mathbf{r})=0 .
$$

Here $\mathbf{k}$ is the momentum conjugate to $\mathbf{r}$ and $E=k^{2} / 2 \mu$ is the energy whereas $\mu$ is the reduced mass. To decouple kinematics from dynamics we make the ansatz:

$\Psi_{\mathbf{k}}(\mathbf{r})=\mathrm{e}^{\mathrm{i} \mathbf{k} \cdot \mathbf{r}} \bar{\Psi}_{\mathbf{k}}(\mathbf{r})$.

The effect of the potential is totally described by the term $\bar{\Psi}$ in Eq. (2). To inspect the asymptotic properties of Eq. (2) we substitute Eq. (2) in Eq. (2) and disregard terms that fall off faster than the Coulomb potential which yields the equation

$\left[-\frac{\mathrm{i}}{\mu} \mathbf{k} \cdot \nabla+\frac{Z_{1} Z_{2}}{r}\right] \bar{\Psi}_{\mathbf{k}}(\mathbf{r})=0$.

This equation can be solved by the ansatz $\bar{\Psi}=\exp (\mathrm{i} \phi)$ which, upon insertion in Eq. (2), leads to the Coulomb-phases

$\phi_{\mathbf{k}}^{ \pm}(\mathbf{r})= \pm \frac{Z_{1} Z_{2} \mu}{k} \ln a(r \mp \hat{\mathbf{k}} \cdot \mathbf{r})$.

The factor $Z_{1} Z_{2} \mu / k$ is called the Sommerfeld parameter and is an indicator for the strength of the interaction. The integration constant $a$ has a dimension of a reciprocal length and is usually set to be $a=k$. The key point for the present work is that the coordinate inherent to Coulomb scattering is the so-called parabolic coordinate $r \mp \hat{\mathbf{k}} \cdot \mathbf{r}$ where the + or - sign should be chosen if one is dealing with incoming or outgoing-wave boundary conditions.

\section{Three-body Coulomb scattering}

For three-body systems the situation is much more complex since the Schrödinger equation is not separable. Nonetheless, as a first step, one might think of a three-body systems as the subsume of three non-interacting two-body subsystems [4-6]. Since we know the appropriate coordinates for each of these two-body subsystems, as illustrated above, the obvious choice of coordinates would be

$$
\left\{\xi_{k}^{\mp}=r_{i j} \pm \hat{\mathbf{k}}_{i j} \cdot \mathbf{r}_{i j}\right\}, \quad \epsilon_{i j k} \neq 0 ; \quad j>i, k \in[1,3],
$$

where $\mathbf{r}_{i j}$ is the position of the particle $i$ relative to the particle $j$ and $\hat{\mathbf{k}}_{i j}$ denote the directions of the momenta $\mathbf{k}_{i j}$ that are conjugate to $r_{i j}$. Since we are dealing with a six-dimensional problem three other independent coordinates are needed in addition to Eq. (5). To make a reasonable choice for these remaining coordinates we remark that, usually, the momenta $\mathbf{k}_{i j}$ are determined experimentally, i.e. they can be considered as the laboratory-fixed coordinates. In fact it can be shown that the coordinates Eq. (5) are related to the Euler angles. Thus, it is advantageous to choose body-fixed coordinates. Those are conveniently chosen as

$\left\{\xi_{k}=r_{i j}\right\}, \quad m \in[4,6]$.

Upon a mathematical analysis it turned out that the coordinates (Eqs. (5) and (6)) are linearly independent [4-6] except for some singular points where the Jacobi determinant vanishes. The main task is now to rewrite the three-body Hamiltonian in the coordinates (Eqs. (5) and (6)). After factoring out the trivial plane-wave part (as done in Eq. 2)) it turns out that the three-body wave function is determined as an eigensolution of an operator $H$ with zero eigenvalue [4-6]. The differential operator $H$, expressed in the curvilinear coordinates (Eqs. (5) and (6)), has the structure

$H=H_{\text {par }}+H_{\text {int }}+H_{\text {mix }}$.

The operator $H_{\mathrm{par}}$ is differential in the parabolic coordinates $\xi_{1,2,3}$ only, whereas $H_{\text {int }}$ acts on internal degrees of freedom $\xi_{4,5,6}$. The mixing term $H_{\text {mix }}$ arises from the off-diagonal elements of the metric tensor and plays the role of rotational coupling in a hyperspherical treatment. The essential point is that the differential operators $H_{\mathrm{par}}$ and $H_{\text {int }}$ are exactly separable in the coordinates $\xi_{1 \ldots 3}$ and $\xi_{4 \ldots 6}$, respectively, for they can be written as [4-6]:

$H_{\mathrm{par}}=\sum_{j=1}^{3} H_{\xi_{j}},\left[H_{\xi_{j}}, H_{\xi_{i}}\right]=0 ; \quad \forall i, j \in\{1,2,3\}$, 
and

$H_{\mathrm{int}}=\sum_{j=4}^{6} H_{\xi_{j}}, \quad\left[H_{\xi_{j}}, H_{\xi_{i}}\right]=0 ; \quad \forall i, j \in\{4,5,6\}$,

where

$H_{\xi_{j}}=\frac{2}{\mu_{l m} r_{l m}}\left[\partial_{\xi_{j}} \xi_{j} \partial_{\xi_{j}}+i k_{l m} \xi_{j} \partial_{\xi_{j}}-\mu_{l m} Z_{l m}\right]$

$\epsilon_{j l m} \neq 0, \quad j \in\{1,2,3\}$

and

$$
\begin{aligned}
& H_{\xi_{4}}=\frac{1}{\mu_{23}}\left[\frac{1}{\xi_{4}^{2}} \partial_{\xi_{4}} \xi_{4}^{2} \partial_{\xi_{4}}+i 2 k_{23} \frac{\xi_{1}-\xi_{4}}{\xi_{4}} \partial_{\xi_{4}}\right], \\
& H_{\xi_{5}}=\frac{1}{\mu_{13}}\left[\frac{1}{\xi_{5}^{2}} \partial_{\xi_{5}} \xi_{5}^{2} 1 \partial_{\xi_{5}}+i 2 k_{13} \frac{\xi_{2}-\xi_{5}}{\xi_{5}} \partial_{\xi_{5}}\right], \\
& H_{\xi_{6}}=\frac{1}{\mu_{12}}\left[\frac{1}{\xi_{6}^{2}} \partial_{\xi_{6}} \xi_{6}^{2} \partial_{\xi_{6}}+i 2 k_{12} \frac{\xi_{3}-\xi_{6}}{\xi_{6}} \partial_{\xi_{6}}\right] .
\end{aligned}
$$

In the equations above the reduced mass of the pair $i j$ is denoted by $\mu_{i j}$ and their product charge by $Z_{i j}$. The operator $H_{\text {mix }}=H-H_{\mathrm{par}}-H_{\mathrm{int}}$ derives from the expression

$$
\begin{aligned}
H_{\text {mix }}:= & \sum_{u \neq v=1}^{6}\left\{\left(\nabla_{\mathbf{r}_{i j}} \xi_{u}\right) \cdot\left(\nabla_{\mathbf{r}_{i j}} \xi_{v}\right)\right. \\
& \left.+\left(\nabla_{\mathbf{R}_{k}} \xi_{u}\right) \cdot\left(\nabla_{\mathbf{R}_{k}} \xi_{v}\right)\right\} \partial_{\xi_{u}} \partial_{\xi_{v}},
\end{aligned}
$$

where $\mathbf{R}_{k}$ indicates the position of the center of mass of the pair $i j$ with respect to the particle $k$.

Noting that $H_{\xi_{j}}, j=1,2,3$ is simply the Schrödinger operator for two-body scattering rewritten in parabolic coordinates (after factoring out the plane-wave part), one arrives immediately, as a consequence of Eq. (8), at an expression for the three-body wave function as product of three two-body continuum waves, provided that $H_{\text {int }}$ and $H_{\text {mix }}$ are negligible. Fortunately, it turns out that the matrix elements of $H_{\text {int }}$ and $H_{\text {mix }}$ are in fact small compared to those of $H_{\mathrm{par}}$ in case of large interparticle separations [4-6]. It should be emphasized, however, that this "asymptotic separability" is not the result of using the coordinate system (5). It is only that the operator $H_{\text {par }}$ attains in the coordinates (5) a very simple and transparent form for an arbitrary three-body system. In fact, this same operator $H_{\text {par }}$ has a much more complex representation in the usual Jacobi coordinates (cf. Refs. [4-6]).

When all particles are close to each other the terms $H_{\text {int }}$ and $H_{\text {mix }}$ becomes more important. In fact, the eigenfunctions of $H_{\mathrm{par}}$ are shown to be at variance (cf. Refs. [4-6]) with the Fock expansion that has to be satisfied at the three-body collision point.

\subsection{Three-body coupling in position space}

The asymptotic properties of the eigenfunctions of $H_{\mathrm{par}}$ are of limited interest when it comes to evaluating reaction amplitudes, for in this case an adequate description of the short-range dynamics is imperative. In fact such amplitudes involve the many-body scattering state in the entire Hilbert space. Moreover, in many processes, the reaction zones are confined to a small region around the origin where all particles are close together. Thus we are obliged to search for solutions that diagonalize, at least, some parts of $H_{\text {int }}$ and $H_{\text {mix }}$ in addition to $H_{\mathrm{par}}$. This can be done in different ways depending on the specific process under considerations and the degree of complexity one is able to handle when calculating numerically the transition amplitudes.

One method that turned out to be particularly effective relies on the following observations: (a) in a three-body system the two-body potential $Z_{i j} / r_{i j}$ have no specific physical meaning since the particles move according the total-potential surface. (b) For this reason the total potential can be split arbitrarily keeping its total value unchanged. (c) To keep the structure of the operators (Eqs. (8) and (10)) unchanged and to introduce a splitting of the total potential that is rotationally invariant one can assume the strength of the individual twobody interactions, characterized by $Z_{i j}$, to be dependent on $\xi_{4,5,6}$. This means we introduce variable product charges as

$\bar{Z}_{i j}=\bar{Z}_{i j}\left(\xi_{4}, \xi_{5}, \xi_{6}\right)$,

with

$\sum_{j>i=1}^{3} \frac{\bar{Z}_{i j}}{r_{i j}}=\sum_{j>i=1}^{3} \frac{Z_{i j}}{r_{i j}}$ 
The simplest way to obtain $\bar{V}_{i j}:=\left(\bar{Z}_{i j}\right) /\left(r_{i j}\right)$ is to assume them as a linear mixing of $V_{i j}:=\left(Z_{i j}\right) /\left(r_{i j}\right)$, i.e.

$$
\left(\begin{array}{l}
\bar{V}_{23} \\
\bar{V}_{13} \\
\bar{V}_{12}
\end{array}\right)=\mathscr{A}\left(\begin{array}{l}
V_{23} \\
V_{13} \\
V_{12}
\end{array}\right),
$$

where $\mathscr{A}\left(\xi_{4}, \xi_{5}, \xi_{6}\right)$ is a $3 \times 3$ matrix. Then the matrix elements have then to be determined according to the properties of the total potential surface and, if possible, as to minimize $H_{\text {int }}$ and $H_{\text {mix }}$. Till now, this has been done for the case of two electrons moving in the field of a residual ion [4-6]. The results for a variety of scattering systems and geometries are encouraging (cf. Ref. [12] and references therein). In Fig. 1(a)-(c) some examples for the electron-impact ionization are depicted that clearly show the importance of three-body coupling as described by Eq. (15).

\subsection{Three-body coupling via momentum exchange}

The obvious shortcoming of the three-body coupling scheme Eq. (15) is that one has to derive $\mathscr{A}$ for each specific scattering system which is a painstaking procedure. An alternative way of coupling the three two-body subsystems relies on the following observations. In a scattering experiment the measurable quantities (observables) are the asymptotic momenta $\mathbf{k}_{i j}$ of the emerging reaction fragments. In the "reaction zone" these quantum numbers are undetermined. To quantify this picture we define, following the treatment of Wannier [16-18], an inner Coulomb zone and an outer far zone depending on whether the total potential or the kinetic energy is the dominant quantity. As is well known [16-18], the boundary between these regimes is the Wannier radius $R_{\mathrm{w}}$ which is a scalar quantity. In the Coulomb zone a two-body subsystem $i j$ can assume any two-body quantum state defined by a particular $\mathbf{k}_{i j}^{\prime}$, i.e. each two-body subsystem propagates off the two-body energy shell, as defined by the (asymptotic) measurement process. The description of this is well facilitated by (Eqs. (8) and (10)) since the momenta $\mathbf{k}_{i j}$ enter in Eq. (10) as dummy parameters and are determined only by the asymptotic boun-
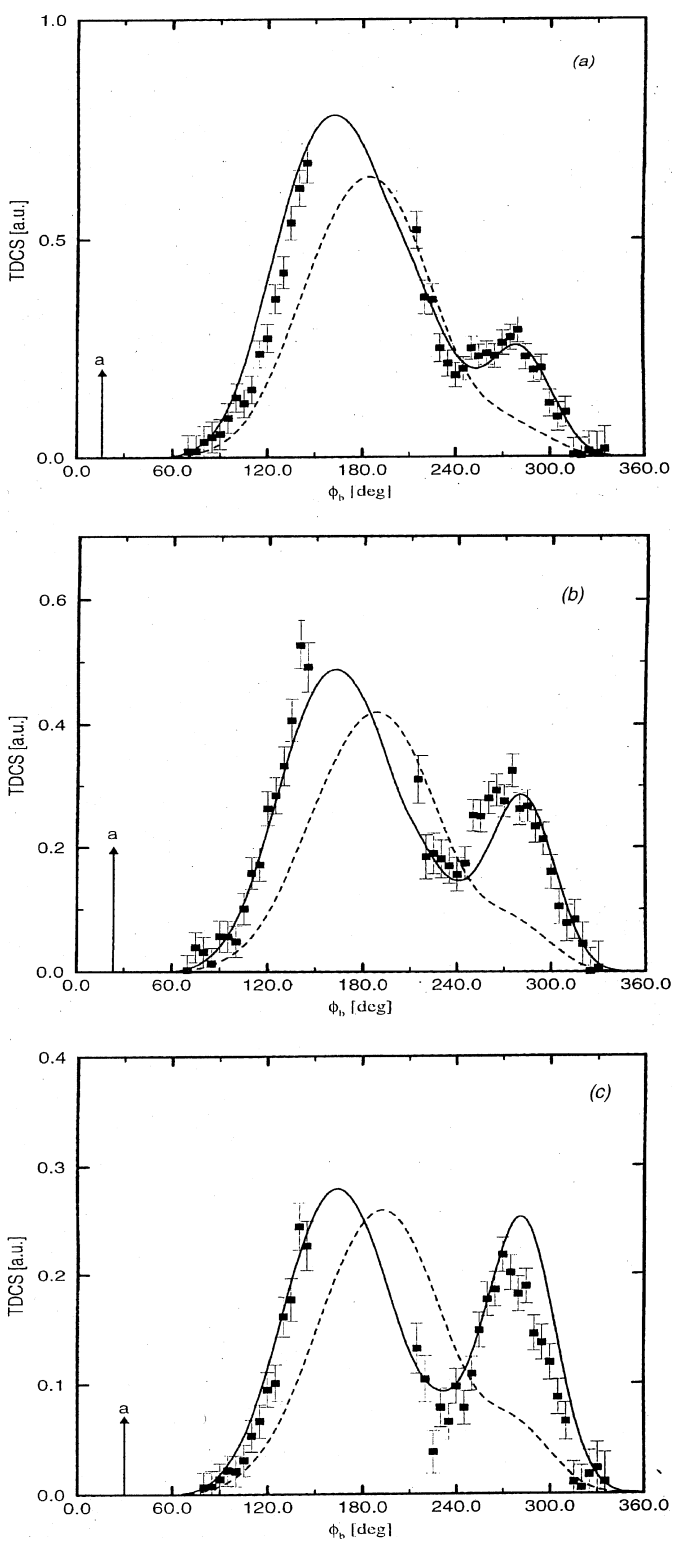

Fig. 1. The fully differential cross section for the electron-impact ionization of atomic hydrogen in the co-planar, asymmetric energy-sharing geometry. The incident energy is $E_{i}=27.2 \mathrm{eV}$. The fast scattered electron is detected at an angle $\Phi_{a}$ with respect to the incident direction $\Phi_{a}=16^{\circ}(\mathrm{a}), \Phi_{a}=23^{\circ}$ (b) and $\Phi_{a}=30^{\circ}$ (c). The angular distribution of the secondary electron with fixed energy of $4 \mathrm{eV}$ is measured. The emission angle of the latter electron with respect to the incident direction is denoted by $\Phi_{b}$. Experiments are courtesy of Ref. [22]. The solid lines show the predictions of the theory employing the matrix $\mathscr{A}$ (cf. Eq. (15)) as given in Ref. [12] whereas the dotted curves indicate the results when using $\mathscr{A} \equiv 1$. 
dary conditions and the total energy conservation. However, to ensure the invariance of the Schrödinger equation under the introduction of intermediate momenta $\mathbf{k}_{i j}^{\prime}$, we must operate under the constraint that the total energy $E$ is conserved. This means that the two-body subsystems exchange an indefinite amount of momentum in the momentum-exchange zone and virtually occupy all (two-body) continuum states available in the energy band $[0, E]$. As the system evolves towards the Wannier boundary $R_{\mathrm{w}}$ the reaction fragments take on the (asymptotic) momenta measured in a scattering reaction. To put the preceding in a mathematical language we note that Eqs. (10) contain the magnitudes of the momenta $k_{i j}$ parametrically. Within the present model, however, the directions $\hat{\mathbf{k}}_{i j}$ are fixed by the (asymptotic) boundary conditions (compare Eqs. 5))]. As mentioned above, the exact normalized eigenfunctions $\Psi_{\text {par }}\left(\xi_{1 \ldots 6} ; \mathbf{k}_{i j}^{\prime}\right)$ of $H_{\text {par }}$ are known and characterized by $\mathbf{k}_{i j}^{\prime}$, or equivalently by $k_{i j}^{\prime 2} / 2 \mu_{i j}=E_{i j}^{\prime} \in[0, E]$. Thus, the general solution in the inner zone is a linear superposition of $\Psi_{\text {par }}\left(\xi_{1 \ldots 6} ; k_{i j}^{\prime}\right)$ :

$$
\begin{aligned}
\Psi^{\mathrm{in}}\left(\xi_{1 \ldots 6}\right)= & \mathscr{N} \int \mathrm{d}^{3} \mathbf{k}_{i j}^{\prime} A_{\mathbf{k}_{i j}^{\prime}}\left(\xi_{4 \ldots 6}\right) \Psi_{\mathrm{par}}\left(\xi_{1 \ldots 6} ; \mathbf{k}_{i j}^{\prime}\right) \\
& \delta\left(E-E^{\prime}\right) \delta^{2}\left(\hat{\mathbf{k}}_{i j}-\hat{\mathbf{k}}_{i j}^{\prime}\right)
\end{aligned}
$$

where $E^{\prime}$ is the intermediate total energy determined by $k_{i j}^{\prime}$. It is readily verified that the function (16) is an eigenfunction of the total Hamiltonian under the approximation $H \approx H_{\text {par }}$. To account for the neglected part $H-H_{\mathrm{par}}=H_{\text {int }}+H_{\text {mix }}$ the expansion coefficients $A_{\mathbf{k}_{i j}^{\prime}}\left(\xi_{4 \ldots 6}\right)$ have to be deduced, e.g. variationally. For many-body continuum states, however, this procedure is intractable. In the context of this work $A_{\mathbf{k}_{i j}^{\prime}}\left(\xi_{4, \ldots 6}\right)$ derives, however, from the observation that the wavefunction (16) is needed only in the reaction zone whose boundary is fixed by $R_{\mathrm{w}}$. The Wannier radius $R_{\mathrm{w}}$, however, scales inversely with $E[17,18]$. Hence, the determination of $A_{\mathbf{k}_{i j}^{\prime}}\left(\xi_{4 \ldots 6}\right)$, and thus of Eq. (16), is needed only at lower excess energies $E$ $\left(\lim _{E \gg 1} R_{\mathrm{w}} \rightarrow 0\right)$. Assuming $H \approx H_{\mathrm{par}}$, we remark further that the expansion coefficients $A_{\mathbf{k}_{i j}^{\prime}}\left(\xi_{4 \ldots 6}\right)$ indicate the occupation probabilities for the intermediate states characterized by $E_{i j}^{\prime} \in[0, E]$. According to the Wannier threshold analysis [16], the correlated motion in the interaction region is ergodic, which implies $A_{\mathbf{k}_{i j}^{\prime}}\left(\xi_{4, \ldots 6}\right)=1$. This means a flat probability distribution of occupying the intermediate virtual states in the inner zone.

The wavefunction (16) is asymptotically incorrect. Hence, a mapping onto the asymptotic wavefunction at the boundary $R_{\mathrm{w}}$ is necessary. Generally, this can be done analogously to the $R$ matrix approach [19]. Here we adopt a different procedure by introducing the exponential matching factor $f:=\exp \left(-R / R_{\mathrm{w}}\right)$ where the extent of the three-body system is measured by $R:=r_{12}+r_{13}$. The wavefunction in the entire Hilbert space is then given by

$$
\Psi_{\mathrm{ex}}\left(\xi_{1 \ldots 6} ; E\right)=f \Psi^{\mathrm{in}}+(1-f) \Psi_{\mathrm{par}}\left(\xi_{1 \ldots 6} ; \mathbf{k}_{i j}\right) .
$$

First we remark that since $R_{\mathrm{w}}$ and $R$ are scalar quantities, i.e., they depend on $\xi_{4 \ldots 6}$ only, the wavefunction $\Psi_{\mathrm{ex}}\left(\xi_{1 \ldots 6} ; E\right)$ is an eigensolution of the total Hamiltonian within the approximation $H \approx H_{\mathrm{par}}=\sum_{j=1}^{3} H_{\xi_{j}}$. As the wavefunction $\Psi_{\text {par }}\left(\xi_{1 \ldots 6} ; \mathbf{k}_{i j}\right)$ is asymptotically correct for large interparticle separations [2,4-6] it follows that these (asymptotic) properties are directly reflected into the wavefunction $\Psi_{\mathrm{ex}}$ ( since $\lim _{R \gg 1} f \rightarrow 0$ ), i.e., $\Psi_{\mathrm{ex}}$ is asymptotically correct for larger interparticle distances. This asymptotic behaviour distinguishes the present study from conventional $R$ matrix methods in which the correct asymptotic behaviour is not easy to include.

For distances $R$ larger than $R_{\mathrm{w}}(f \rightarrow 0)$ we obtain $\Psi_{\text {ex }}=\Psi_{\text {par }}\left(\xi_{1 \ldots 6} ; \mathbf{k}_{i j}\right)$ which means that in the high-energy scattering $\left(R_{\mathrm{w}} \rightarrow 0\right)$ the escaping particles directly assume their experimentally measured momenta. For $R<R_{\mathrm{w}}$ the two-body subsystems exchange an indefinite amount of energy. Of particular interest is the region of low excess energies where $R_{\mathrm{w}}$ extends to very large distances $R$, i.e., $f \rightarrow 1$. In this case the three particles keep exchange energies up to infinity for $E \rightarrow 0$ and the transformation of the total wavefunction from $\Psi^{\text {in }}$ to $\Psi_{\text {par }}$ occurs at very large distances. This has important implications in so far as, according to Eq. (17), properties of scattering amplitudes which are derived from asymptotic wavefunctions are smeared out at threshold. 
A second important conclusion at threshold is that if we extend the integral in Eq. (17) to run over bound states as well, the coupling to highly excited Rydberg states provides the major contribution to the wavefunction, given by Eq. (17), in agreement with the Wannier picture of double escape [16-18].

The above formulation can also be done directly for the $T$-matrix as shown in Ref. [15]. As an application of this method let us study the proton and antiproton-impact ionization of atomic hydrogen that leads to a pure three-body continuum final state. The heavy projectile is mainly scattered into the forward direction and different ionization mechanisms are distinguished via the particles' relative velocities (since $Z_{i j}= \pm 1$ ). The most notable difference between proton and antiproton impact appears at a diminishing relative velocity vector $\mathbf{v}_{\mathrm{pe}}$ of the projectile-electron system (Fig. 2(a)). This is due to the decisively different analytical behavior of the projectile-electron density of state which, for $v_{\text {pe }} \rightarrow 0$, is of the form $\exp \left(-1 / v_{\text {ep }}\right) \rightarrow 0$ for antiproton and $1 / v_{\text {pe }} \rightarrow \infty$ for proton impact $[20,21]$.

The ridge structure in Fig. 2(a) which appears at an electron velocity equal to twice the projectile's velocity (in the target frame) is due to a direct projectile-electron encounter [20]. Energy exchange effects are prominent in the region where a high-energy electron is ejected backwards (Fig. 2(b)). In this case the electron can not be viewed as emitted in the field of the target (slow soft electrons) nor in the field of the projectile (Electrons Captured into the projectile's Continuum, ECC electrons with $v_{\mathrm{pe}} \rightarrow 0$ ). Further inspection showed that these electrons are ejected via multiple scattering from both the target nucleus and the projectile in events with large deflection of the projectile.

The approaches sketched above can be generalized to $N$-body systems [13] with increasing complexity of the mathematical analysis.

For highly excited, yet still bound systems different mathematical tools are necessary [14]. Nonetheless it turned out that a number of common features of the system persist when crossing the fragmentation threshold.
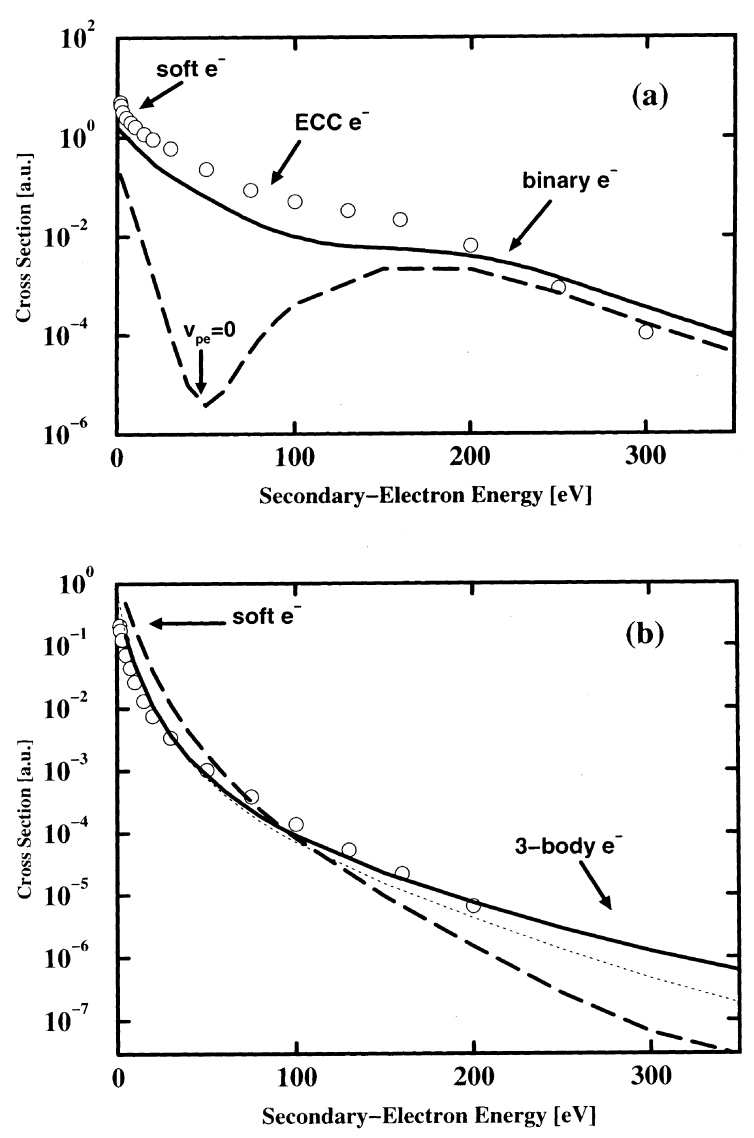

Fig. 2. The energy spectrum of secondary electrons ejected from atomic hydrogen upon proton (solid curve) and antiproton (dashed curve) impact with incident energy of $95 \mathrm{keV}$. In (a) the electrons are detected at an angle of $15^{\circ}$ with respect to the incident beam whereas in (b) this angle is fixed to $150^{\circ}$. The calculations have been performed using the wave function Eq. (17) for the description of the final state. In (b) calculations using $\Psi_{\mathrm{ex}} \approx \Psi_{\mathrm{par}}$ are included (dotted curve). The absolute experimental data are due to Ref. [23] where comparison with other theories can be found.

\section{References}

[1] L. Rosenberg, Phys. Rev. D 8 (1973) 1833.

[2] M. Brauner, J.S. Briggs, H. Klar, J. Phys. B 22 (1989) 2265.

[3] E.O. Alt, A.M. Mukhamedzhanov, Phys. Rev. A 47 (1993) 2004.

[4] J. Berakdar, Phys. Rev. A 53 (1996) 2314.

[5] J. Berakdar, Phys. Rev. A 54 (1996) 1480.

[6] J. Berakdar, Phys. Lett. A 220 (1996) 237. 
[7] Sh.D. Kunikeev, V.S. Senashenko, Zh. E’ksp. Teo. Fiz. 109 (1996) 1561.

[8] Sh.D. Kunikeev, V.S. Senashenko, Sov. Phys. JETP 82 (1996) 839

[9] D.S. Crothers, J. Phys. B 24 (1991) L39.

[10] E.O. Alt, A.M. Mukhamedzhanov, Phys. Rev. A 47 (1993) 2004.

[11] G. Gasaneo, F.D. Colavecchia, C.R. Garibotti, J.E. Miraglia, P. Macri, Phys. Rev. A 55 (1997) 2809.

[12] J. Berakdar, Phys. Rev. A 56 (1997) 370.

[13] J. Berakdar, Phys. Rev. A 55 (1997) 1994.

[14] J. Berakdar, Phys. Rev. A 58 (1998) 1641.
[15] J. Berakdar, Phys. Rev. Lett. 78 (1997) 2712.

[16] G. Wannier, Phys. Rev. 90 (1953) 817.

[17] A.R.P. Rau, Phys. Rev. A 4 (1971) 207.

[18] A.R.P. Rau, Phys. Rep. 110 (1984) 369.

[19] K.A. Berrington, P.G. Burke, K. Butler, M.J. Seaton, P.J. Storey, K.T. Taylor, Yu Yan, J. Phys. (1987) 6379.

[20] P.D. Fainstein, V.H. Ponce, R.D. Rivarola, J. Phys. B 24 (1991) 3091.

[21] J. Berakdar, J.S. Briggs, H. Klar, Z. Phys. D 24 (1992) 351.

[22] J. Röder, private communication.

[23] G.W. Kerby III, M.W. Gealy, Y.Y. Hsu, M.E. Rudd, Phys. Rev. A 51 (1995) 2256. 